\title{
Effect of oxygen surfactant on the magnetic and structural properties of Co films grown on $\mathrm{Cu}(110)$
}

\author{
W. L. Ling, Z. Q. Qiu \\ Department of Physics, University of California, Berkeley, California 94720 \\ O. Takeuchi,* D. F. Ogletree and M. Salmeron \\ Materials Sciences Division, Lawrence Berkeley National Laboratory, \\ University of California, Berkeley, California 94720
}

\begin{abstract}
It was found that atomically flat $\mathrm{Co}(110)$ films could be grown on $\mathrm{Cu}(110)$ using $\mathrm{O}$ as a surfactant. To obtain detailed knowledge on the effect of $\mathrm{O}$ on the growth, as well as on the magnetic properties of $\mathrm{Co}$ overlayer, we carried out an investigation on this system using Auger Electron Spectroscopy (AES), Low Energy Electron Diffraction (LEED), Surface Magneto-Optic Kerr Effect (SMOKE), and Scanning Tunneling Microscopy (STM). With O as a surfactant, the initial growth of Co $(<1 \mathrm{ML})$ results in a flat monolayer structure. When the Co is thicker than $1 \mathrm{ML}$, three-dimensional clusters begin to form. These clusters become ordered islands at $3 \mathrm{ML}$ Co and coalesce at $~ 5$ ML Co. Above 5 ML Co, layer-by-layer growth resumes. No significant $\mathrm{Cu}$ segregation is observed. SMOKE studies at room temperature show that the Co film is magnetic above $\sim 5 \mathrm{ML} \mathrm{Co}$, with the magnetization easy axis along the [001] direction. On the other hand, without using oxygen as a surfactant, Co grows three-dimensionally on $\mathrm{Cu}(110)$. The Co overlayer has its easy magnetization axis along the [001] direction, but the onset of the magnetization was observed at $11 \mathrm{ML}$ Co at room temperature.
\end{abstract}

PACS numbers: 75.70.ak, 75.30.kz, 75.30.pd,75.50.Bb

\section{INTRODUCTION}

$\mathrm{Co} / \mathrm{Cu}$ heterostructures have attracted significant interest since the discovery of oscillatory magnetic interlayer coupling ${ }^{1}$ and the giant magnetoresistance (GMR). ${ }^{2}$ Many magnetic properties, such as 
interlayer coupling, giant magnetoresistance, Curie temperature $\left(\mathrm{T}_{\mathrm{c}}\right)$ and magnetic anisotropy, etc., have been intensively investigated and were found to have a strong dependence on the interfacial property and the film quality. ${ }^{3,45,5,7}$ Compared to the many studies of $\mathrm{Co}$ grown on $\mathrm{Cu}(100)$ and $\mathrm{Cu}(111)$, less effort has been made on the $\mathrm{Co} / \mathrm{Cu}(110)$ system because of the three-dimensional (3D) growth nature on the lowsymmetry (110) surface. $^{8}$ On the other hand, the low-symmetry surface generates an in-plane magnetic anisotropy, ${ }^{9}$ which can be used to address a number of fundamental issues in low-dimensional magnetic systems. ${ }^{10}$ For example, the (110) orientation allows differentiation between cubic and uniaxial anisotropy contributions due to different symmetry axes. ${ }^{8}$ Since the (110) plane contains the three major symmetry axes-[111], [001] and [1-10]—a study of the magnetic properties of this system further provides us with the opportunity of manipulating the magnetization direction along different crystal axes. In fact, a sudden breakdown of magnetocrystalline anisotropy (MCA) occurs for Co films below $50 \AA^{11}$ on $\mathrm{Cu}(110)$ and the easy magnetization axis lies in-plane along the [001] direction. ${ }^{12,13}$ More interestingly, a switch of the easy magnetization axis with a slight dose of $\mathrm{CO}$ from the [001] to the [1-10] direction was recently observed and can be reversed by depositing a sub-monolayer of $\mathrm{Cu}^{14}$ A large part of the observed magnetic anisotropy behavior is attributed to the strain and morphology of the film. This has been investigated by low energy electron diffraction (LEED) and scanning tunneling microscopy (STM), which revealed anisotropic island growth leading to anisotropically relaxed strain. ${ }^{12,15}$

Theoretical works have also been carried out for this system. First-principles calculations using fullpotential linearized-augmented-plane wave (FLAPW) method predicts that the easy axis for a monolayer fcc $\operatorname{Co}(110)$ film lies in-plane along the $[1-10]$ axis $^{16}$ and a realistic tight-binding model for fcc (110) Co slabs gives forth to an in-plane magnetization of direction varying with slab thickness. ${ }^{17}$ A more recent theoretical work, using the spin-polarized relativistic linear muffin-tin orbital (SPR-LMTO) method, yields results that most closely match experimental results. ${ }^{18}$ The uniaxial strains not only induce large uniaxial and planar magnetoelastic anisotropies, but can also act to suppress the cubic MCA. Nonetheless, the MCA constant $\mathrm{K}_{1}$ from these calculations remains more or less constant within the misfit isotropic in-plane strains that are possibly in the fcc $\mathrm{Co}(110)$ films on $\mathrm{Cu}(110)(0-2 \%)$. No abrupt transformation around 50 $\AA$ layer thickness was found. The suppression observed in the experiment may therefore be due to the 
change in the growth pattern, instead of the increase in the isotropic in-plane strain. As considerable Co-Cu intermixing is present in this system, the discrepancy may also have come from the interaction between Co and $\mathrm{Cu}^{8}{ }^{8}$ While the Co-Cu interaction was ignored in the SPR-LMTO calculations, there has been evidence showing that it may play a significant role in the in-plane MCA. ${ }^{16,19}$ This problem would be simplified and better understood if the 3D growth mode and $\mathrm{Co}-\mathrm{Cu}$ intermixing in the $\mathrm{Co} / \mathrm{Cu}(110)$ system could be circumvented. Therefore, it is highly desirable to attain a flat $\mathrm{Co} / \mathrm{Cu}(110)$ film experimentally in order to gain a thorough understanding of the magnetic anisotropy in this system.

A recent study applying helium scattering oscillations showed that using oxygen as a surfactant leads to atomically flat $\mathrm{Co}(110)$ films grown on $\mathrm{Cu}(110) .{ }^{20}$ Our STM results demonstrated that a well-ordered two-dimensional surface initially forms at $1 \mathrm{ML}$ Co coverage on the saturated $\mathrm{Cu}-(2 \times 1) \mathrm{O}$ surface ${ }^{21}$, which may play a role in the subsequent growth. On the other hand, it has been reported that dosing oxygen destroys the magnetism of Co films. ${ }^{13}$ Therefore, it would be very useful to have a comprehensive study of the effect of an oxygen surfactant on the growth and magnetic properties of $\mathrm{Co}$ grown on $\mathrm{Cu}(110)$. In this paper, we present such a study of the $0-30 \mathrm{ML}$ range of Co films grown at $\sim 350 \mathrm{~K}$ on the $\mathrm{Cu}-(2 \times 1) \mathrm{O}$ surface, compared to Co films in the same thickness range grown under the same conditions on the bare $\mathrm{Cu}(110)$ surface. Structural properties were obtained using Low Energy Electron Diffraction (LEED), Reflection High Energy Electron Diffraction (RHEED), Auger Electron Spectroscopy (AES), and Scanning Tunneling Microscopy (STM). Surface magneto-optical Kerr effect (SMOKE) was used to measure the magnetic properties. We found that the oxygen surfactant resulted in an initial 3D growth between $1 \mathrm{ML}$ Co and $\sim 5 \mathrm{ML}$ Co, and then led to a quasi layer-by-layer growth until $\sim 25 \mathrm{ML}$, when the lattice misfit started to relax the strain of the film. The resulting film was free of $\mathrm{Cu}$, with the oxygen floating on top. In agreement with this outcome, SMOKE at room temperature shows an onset of magnetic order at $\sim 5 \mathrm{ML}$ Co. In contrast, $\mathrm{Co}$ film grown on bare $\mathrm{Cu}(110)$ with the same growth conditions showed 3D growth and room temperature onset of magnetization occurred at $\sim 11$ ML Co.

\section{EXPERIMENT}

The experiments were performed on a $\mathrm{Cu}(110)$ substrate in an ultrahigh vacuum (UHV) system equipped with quadrupole mass spectrometer (QMS), LEED, RHEED, AES, STM, and SMOKE. The 
pressure of the system was in the $10^{-10}$ Torr range and rose into the low $10^{-9}$ Torr range during the Co film growth. Co was evaporated from an alumina crucible wrapped with a tungsten filament while the evaporation rate was monitored by a quartz crystal oscillator located near the $\mathrm{Cu}$ crystal. The typical $\mathrm{Co}$ evaporation rate was $\sim 0.5-0.8 \mathrm{ML} / \mathrm{min}$, corresponding to a crucible temperature of $\sim 100{ }^{\circ} \mathrm{C}$.

The $\mathrm{Cu}(110)$ substrate was cleaned in vacuum with Ar ion sputtering and annealing cycles until a sharp $(1 \times 1)$ LEED pattern was observed. Surface cleanliness was checked by AES. After cleaning, the surface was exposed to $\sim 10 \mathrm{~L}$ of oxygen at a temperature of $600 \mathrm{~K}$ to form the $\mathrm{Cu}(110)-(2 \times 1) \mathrm{O}$ surface. ${ }^{22,23}$ The substrate was then cooled for about $10 \mathrm{~min}$ in $5 \times 10^{-8}$ Torr oxygen atmosphere. Subsequently the Co overlayer, either as a flat uniform film or as a wedge, was grown on top of the $\mathrm{Cu}(110)-(2 \times 1) \mathrm{O}$ structure at a substrate temperature of $350 \mathrm{~K}$. The wedge was created by translating the substrate at a constant speed behind a knife edge shutter and the slope of the wedge was determined by the evaporation rate together with the sample moving speed. A typical wedge had a slope of $\sim 6 \mathrm{ML} / \mathrm{mm}$. At the end of the wedge, a thick Co layer ( 40-50 ML) was grown for the purpose of wedge registration using SMOKE. Fig. 1 shows a schematic drawing of the sample. For comparison, we also prepared a sample of the same Co wedge grown on a clean $\mathrm{Cu}(110)$ crystal under identical conditions with substrate temperature of $350 \mathrm{~K}$, except for the oxygen treatment.

Structural properties of the films were characterized by AES, LEED, RHEED and STM at room temperature. LEED and RHEED measurements were performed along the Co wedge for different Co thickness. For STM studies, uniform Co films were grown due to the finite scanning range of the STM. An etched Pt-Rh alloy tip was used for imaging. Images shown in this paper are topographic images taken at constant tunneling current with the tip biased. The STM experimental details are further discussed in an earlier paper describing the first monolayer growth of $\mathrm{Co}$ on the $\mathrm{Cu}(110)-(2 \times 1) \mathrm{O}$ surface. ${ }^{21}$

SMOKE measurements were performed on the Co wedges at room temperature. A He-Ne laser was used as the light source for the SMOKE experiments. The incident beam was p-polarized and was at an angle of incidence of $45^{\circ}$. Two pairs of electromagnets generated a magnetic field either perpendicular or parallel to the film surface so that both polar and longitudinal hysteresis loops could be obtained without having to move the sample. The intensity of the reflected beam from the sample surface was measured 
with a photodiode as a function of the applied magnetic field. A $1 / 4$ waveplate and a polarizer were placed in front of the photodiode, with the polarizer $1^{\circ}$ away from the extinction. For all the films measured in this work, magnetization was in the plane of the film as no polar remenance was detected. Hence, all hysteresis loops shown in this paper are from longitudinal measurement. By rotating the sample, longitudinal magnetization could be made along both the [001] and [1-10] directions.

\section{RESULTS AND DISCUSSION}

\section{A. AES}

AES results on the growth of $\mathrm{Co}$ on the $\mathrm{Cu}(110)-(2 \times 1) \mathrm{O}$ surface were reported in an earlier paper. ${ }^{21}$ To make this paper self-contained, we summarize here the AES results in Fig. 2. The oxygen peak intensity was nearly invariant as a function of the Co layer thickness, showing that oxygen served as a surfactant in this system, i.e., the oxygen atoms remained at the top of the film as Co thickness increases. While Co AES signals increased with Co thickness, $\mathrm{Cu}$ AES signals decreased to approach zero. This result shows that except perhaps during the first or second monolayers, little or no alloying or segregation occurred in this system. This is contrary to the strong agglomeration and/or segregation of $\mathrm{Cu}$ atoms for $\mathrm{Co}$ grown on clean $\mathrm{Cu}(110)^{8}$.

\section{B. SMOKE}

SMOKE measurements were done from 0 to $30 \mathrm{ML}$ of $\mathrm{Co}$ along the wedge grown on the $\mathrm{Cu}(110)-(2 \times$ 1)O surface. Fig. 3 shows the longitudinal hysteresis loops of Co films at various thickness with the magnetic field applied along the [001] and [1-10] crystal axes, respectively. The magnetic remanence onset appears at $\sim 5 \mathrm{ML}$ Co. Since the SMOKE measurements were taken at room temperature, the disappearance of the magnetization below $5 \mathrm{ML}$ Co could be due to a drop in the Curie temperature to below room temperature or a super-paramagnetic regime with a blocking temperature lower than the room temperature. As $5 \mathrm{ML}$ Co is the thickness where the transition of 3D to layer-by-layer growth occurs from the He diffraction experiment, ${ }^{20}$ it is likely that the Co islands below $5 \mathrm{ML}$ are super-paramagnetic and are too small to give a blocking temperature higher than room temperature. Above $5 \mathrm{ML}$, the Co islands coalesce into a continuous film so that non-zero magnetic remanence appears. Although we have no rigorous proof of this, magnetic measurements for $\mathrm{Co}$ films grown on clean $\mathrm{Cu}(110)$ and their structural 
characterization by LEED and STM (see discussions later in this paper) support the assertion that the disappearance of the magnetization below 4 ML Co is due to the super-paramagnetic nature of ensembles of Co islands, whose size is too small to give a blocking temperature higher than the room temperature. Another indirect evidence is that $\mathrm{Co}$ films grown on $\mathrm{Cu}(001)$ have a Curie temperature higher than room temperature above $\sim 2 \mathrm{ML} \mathrm{Co}$, and the Curie temperature increases rapidly with Co thickness $(\sim 200$ $\mathrm{K} / \mathrm{ML}){ }^{24}$ Based on this fact, it would be surprising if the disappearance of the magnetic remanence near 5 $\mathrm{ML}$ of $\mathrm{Co}$ on $\mathrm{Cu}(110)-(2 \times 1) \mathrm{O}$ were due to a Curie temperature below room temperature.

Fig. 3 also shows the anisotropic behavior of the hysteresis loops. The full magnetic remanence for magnetic field applied along the [001] direction and zero remanence for field along the [1-10] direction show that the easy magnetization direction is along the [001] direction. In the following, we shall examine the cause of this magnetic anisotropy. First, volume magnetic anisotropy, which is the lowest order of magnetic anisotropy in bulk materials, could give rise to an easy axis at some angle between the [001] and [1-10] directions. However, noting that $\mathrm{fcc} \mathrm{Co} / \mathrm{Cu}(001)$ films show in-plane magnetization with the easy magnetization axis along the [1-10] rather than the [100] direction ${ }^{25}$ and that (001) fcc film does not carry in-plane uniaxial magnetic anisotropy, the volume anisotropy of fcc Co should not be responsible for the observed [001] easy magnetization axis in the $\mathrm{Co} / \mathrm{Cu}(110)-(2 \times 1) \mathrm{O}$ system. Second, uniaxial anisotropy could be generated in these thin films by symmetry breaking at the surface or by strain. For the (110) surface, in particular, the two-fold rotational symmetry could result in an in-plane uniaxial anisotropy. We believe that the observed in-plane uniaxial magnetic anisotropy is due to this symmetry breaking.

For comparison, SMOKE measurements were also performed on the Co films grown on the clean $\mathrm{Cu}(110)$ surface without using oxygen surfactant. The hysteresis loops at various Co thicknesses are shown in Fig. 4. As in Fig. 3, the easy magnetization axis is along the [001] direction. However, in this case, the onset of the magnetic Kerr signal appeared at $\sim 11 \mathrm{ML}$ of Co. To better view the difference in the onset magnetization, we plotted the magnetic remanence in Fig. 5 as a function of Co film thickness for Co on $\mathrm{Cu}(110)-(2 \times 1) \mathrm{O}$ and clean $\mathrm{Cu}(110)$, respectively. Note that the slopes of the two cuves are different. The comparable SMOKE signals of the films with and without oxygen surfactant at $~ 30 \mathrm{ML} \mathrm{Co,} \mathrm{and} \mathrm{the}$ fact that figure $5 \mathrm{~b}$ is not a simple shift of figure $5 \mathrm{a}$ by $\sim 11 \mathrm{ML}$ indicate that the first 11 ML Co grown on 
clean $\mathrm{Cu}(110)$ is not magnetically dead for thicker films and that the disappearance of the magnetic remanence at $10 \mathrm{ML}$ Co is due to super-paramagnetism with a blocking temperature below room temperature. The dramatic difference in the onset magnetization shows that the oxygen surfactant has a significant effect on improving the quality of the Co film. Next we will discuss the effect of the oxygen surfactant on the growth of $\mathrm{Co}$ on $\mathrm{Cu}(110)$.

\section{LEED and RHEED}

LEED and RHEED studies were done on the wedged samples grown on $\mathrm{Cu}(110)-(2 \times 1) \mathrm{O}$ and clean $\mathrm{Cu}(110)$ surfaces, respectively. The energy of the electron was $105 \mathrm{eV}$ for LEED and the electron beam averaged over a thickness range of $\sim 4 \mathrm{ML}$ on the wedge due to the finite width of the LEED beam. RHEED patterns were taken with a $10 \mathrm{keV}$ electron beam parallel to the [001] direction. The LEED and RHEED results are summarized in Figs. 6 and 7.

Fig. 6(a) shows the LEED patterns for Co films grown on $\mathrm{Cu}(110)-(2 \times 1)-\mathrm{O}$ surface. The sharpness and brightness of the LEED pattern for the $\mathrm{Cu}(110)-(2 \times 1)-\mathrm{O}$ surface indicated a well-ordered and smooth starting surface for growth. After 0 - 3 ML Co deposition, the LEED background intensity increased due to diffuse scattering. In addition to the $\left(\_, 1 / 2\right)$ order LEED spots corresponding to the $(2 \times 1)$ reconstruction, new $\left(1 / 2,{ }_{-}\right)$spots could be seen. The diffuse background indicates that the surface had become rougher and was less well-ordered. The diffuse background of the LEED pattern increased in intensity with increasing Co thickness until 3 - 6 ML Co coverage, at which point only the substrate spots remained visible. Beyond $\sim 5$ ML Co, coinciding with the onset of magnetization in Fig. 3, the LEED background dropped. Moreover, streaking developed along [1-10] direction with faint spots half way and a third of the way between the substrate spots. A $(3 \times 1)$ structure was reported by Tölkes et al. for 9.7 ML Co film in this system, ${ }^{20,26}$ but no surface structure giving rise to $\left(\_, 1 / 2\right)$ spots has been reported previously. For Co film thicker than $20 \mathrm{ML}$, spots along the [1-10] direction became more visible but the substrate spots broadened. At $\sim 30$ ML Co coverage, the $\mathrm{p}(1 \times 1)$ spots were visibly broader and more diffuse.

The information extracted from LEED was generally reproduced in the RHEED studies (see Fig. 6(b)). The sharp RHEED streaks for the $\mathrm{Cu}-\mathrm{O}$ surface indicated a good quality surface. After $1 \mathrm{ML}$ of $\mathrm{Co}$, the surface became rough and disordered, as indicated by the broadened RHEED streaks and diffuse 
background. The $(2 \times 1)$ reconstruction from the original $\mathrm{Cu}-\mathrm{O}$ surface had disintegrated and the crystallinity of the surface was poor. This persisted up to $5 \mathrm{ML}$ of Co. Above this thickness, the surface began to re-establish order and $3 \mathrm{x}$ and $2 \mathrm{x}$ periodicities along the [1-10] direction appear. At 20 ML Co, the $3 x$ periodicity seemed to be prevalent while at $30 \mathrm{MLCo}$, the $2 \mathrm{x}$ periodicity appears to be dominant on the surface.

Co films grown on a clean $\mathrm{Cu}(110)$ surfaces showed very different results. LEED patterns for Co films of various thickness are shown in Fig. 7(a). Co grew epitaxially but without additional-long range order, as indicated by the $\mathrm{p}(1 \times 1)$ pattern. The broad spots and diffuse background suggest poor surface ordering. As Co thickness increased, the background intensity increased. Concurrently, the primary spots became weaker and broader, and faint streaks developed along both the [001] and [1-10] directions. Some weak spots were barely visible along the streaks showing weak surface reconstruction. These patterns are different from those reported in ref. 12. Islands elongated in the [1-10] [ref. 12] or the [100] [ref. 15] direction have been observed for $\mathrm{Co}$ grown on clean $\mathrm{Cu}(110)$ at room temperature. Such elongation could have come from anisotropic diffusion or from growth at off-normal incidence, in which case the aspect ratio depends sensitively on the substrate temperature. ${ }^{27}$ The different LEED patterns we obtained may be due to the higher substrate temperature in our experiments. The RHEED patterns (Fig. 7(b)) for this sample show that the streaks broke into spots, further confirming the poor ordering in the growth. This result is consistent with 3D growth which was suggested by the fact that the onset of magnetization in $\mathrm{Co} / \mathrm{Cu}(110)$ occurred at a much higher thickness than the $\mathrm{Co} / \mathrm{Cu}(110)-(2 \times 1) \mathrm{O}$ system.

We will now summarize the structure and morphology of the $\mathrm{Co}$ films grown on the $\mathrm{Cu}-\mathrm{O}$ surface and the bare $\mathrm{Cu}$ surface from the LEED and RHEED results. Above $5 \mathrm{ML}$, Co films grown on the $\mathrm{Cu}-\mathrm{O}$ surface were pseudomorphic with an ordered reconstructed surface. In contrast, Co films grown on clean $\mathrm{Cu}(110)$ showed poor ordering throughout the thickness range. The oxygen surfactant thus significantly improved the quality of the Co film grown on $\mathrm{Cu}(110)$.

\section{STM}

As suggested in the LEED and RHEED section above, the first monolayer growth of Co on the $\mathrm{Cu}(110)-(2 \times 1) \mathrm{O}$ surface involved extensive reconstruction of the surface. This has been confirmed by our 
early STM studies in this thickness regime. ${ }^{21}$ We will briefly summarize our STM results of the first monolayer $\mathrm{Co}$ growth in the following. The initial $\mathrm{Cu}(110)-(2 \times 1)-\mathrm{O}$ structure consists of $\mathrm{Cu}-\mathrm{O}$ chains along the [001] direction. $\mathrm{Cu}$ atoms along these chains are located in the same positions as on the $\mathrm{Cu}(110)$ surface, but the separation distance between adjacent $\mathrm{Cu}-\mathrm{O}$ chains is twice the $\mathrm{Cu}$ lattice spacing along the [1-10] direction. This surface structure gives the $(2 \times 1)$ LEED pattern in Fig. 6(a). At low Co coverage $(<0.25 \mathrm{ML})$, Co atoms on the $\mathrm{Cu}(110)-(2 \times 1) \mathrm{O}$ surface exchange with the $\mathrm{Cu}$ atoms in the $\mathrm{Cu}-\mathrm{O}$ chains to form an alloyed $\mathrm{CuCo}-(2 \times 1)-\mathrm{O}$ structure. The displaced $\mathrm{Cu}$ atoms and additional $\mathrm{Co}$ atoms combine with oxygen atoms to form islands with the same CuCo- $(2 \times 1)$-O structure. The surface then consists of islands scattered over the original flat surface, with the alloyed $\mathrm{CuCo}-(2 \times 1)-\mathrm{O}$ structure on the top layer. Starting from 0.25 ML Co coverage, a new structure starts to form, which consists of $\mathrm{CoCuO}$ rows running along the [1-10] direction. These rows have an internal periodicity twice the $\mathrm{Cu}$ lattice spacing along the [1-10] direction and adjacent rows are separated by two $\mathrm{Cu}$ lattice spacings along the [001] direction. The corrugation between adjacent rows, however, is randomly in-phase or out-of-phase, i.e., the resulting structure is short-ranged and consists of randomly mixed $(2 \times 2)$ and $\mathrm{c}(2 \times 4)$ domains. This new structure is responsible for the additional LEED spots along the [001] direction observed in the 0 -3 ML Co LEED picture (Fig. 6(a)).

Between 1-5 ML Co coverage, the growth becomes 3D. 3D clusters start growing on the monolayer structure beyond 1 ML Co. An STM image at this stage is shown in Fig. 8, which was taken at 1.7 ML Co deposition. Clusters had covered almost the whole lower surface with the height of the clusters ranging from 0.85-4.25 ̊.. At $\sim 3$ ML Co deposition (Fig. 9), the clusters had grown into rectangular islands of dimensions $\sim 45 \AA$ along the [001] direction and $\sim 30 \AA$ along the [1-10] direction, with a center-to-center separation of $\sim 55 \AA$. Their height ranged from $2 \mathrm{ML}$ above the $(2 \times 2)$ surface for smaller islands to $6 \mathrm{ML}$ high for larger islands, with 5 ML the most common height. The formation of these clusters and islands explains the diffused LEED background in this thickness range. As for the surface of these islands, areas with different types of structure were observed. The most prominent type was a $(3 \times 1)$ row structure with rows running along the [001] direction. These rows had inter-row spacing three times the $\mathrm{Cu}$ lattice spacings. Corrugation across the rows was $\sim 0.27 \AA$ A, but periodicity along the rows could not be resolved. 
Since these islands were nucleated randomly at the surface, no obvious phase relations existed between neighboring islands. The region between the islands was still covered by the monolayer structure at $1 \mathrm{ML}$.

With further Co deposition, the islands grew laterally in size and started to merge at $\sim 5 \mathrm{ML}$ of Co coverage (Fig. 10). Meanwhile, the surface of the film developed a 'checkerboard'-like pattern, but retaining the same types of structure as those at 3 ML Co. The height of most of the islands seen in Fig. 10 was $6 \mathrm{ML}$, with a few being $8 \mathrm{ML}$. The center-to-center distance between these islands was still $\sim 55 \AA$. Nonetheless, due to the lateral growth of the islands, the space among the islands was greatly reduced compared to that in the $3 \mathrm{ML}$ Co film. We believe that it is the coalescence of these flat islands that sharpens the LEED spots for Co coverage above $5 \mathrm{ML}$ and locks the super-paramagnetism of the islands to give an onset Kerr signal. Beyond $5 \mathrm{ML} \mathrm{Co,} \mathrm{most} \mathrm{of} \mathrm{the} \mathrm{islands} \mathrm{had} \mathrm{coalesced} \mathrm{into} \mathrm{a} \mathrm{continuous} \mathrm{film} \mathrm{that}$ increased in thickness. Fig. 11 shows an STM image with $10 \mathrm{ML}$ of Co coverage. The variation in height across the film surface was only $\sim 2-3 \mathrm{ML}$, indicating the formation of a flat film that is in agreement with the He diffraction results. ${ }^{20}$ The surface at this stage consists mostly of $(3 \times 1)$ patches, which were about $\sim 25 \AA$ along the [1-10] direction and $\sim 65 \AA$ along the [001] direction. These patches came from the individual islands before coalescence. Because of the random phases between the patches, the $(3 \times 1)$ structure was only barely visible in the 10 ML Co LEED pattern. The numerous anti-phase boundaries among these islands contributed to the streaking in the [1-10] direction in the LEED patterns of this thickness range. Among the patches, there were regions that were not well resolved, as well as holes that were 3-6 ML deeper than the surface. (This is discussed in more detail later in this section.) Further increasing the Co coverage did not change the surface morphology compared to the 10 ML Co film, but the $(3 \times 1)$ patches had noticeably increased in size along the [001] direction, giving a clear $(3 \times 1)$ LEED pattern in this thickness range. Fig. 12 shows an STM image of the film with 19 ML Co.

The system continued this quasi layer-by-layer growth until the film relaxed from the stress accumulated due to the lattice misfit. Fig. 13 shows the surface of the film with 24 ML Co. A number of edge dislocations along the [1-10] direction could be seen. Since the miscut angle of this crystal was close to the [1-10] axis, the steps on the crystal surface might have contributed to releasing the stress in the [001] direction. 
We will now discuss the regions not covered by the $(3 \times 1)$ structure in the STM images. These regions consisted of areas that were less well-ordered and also holes that were several monolayers deeper than the surface. The less well-ordered areas consisted of short pieces separated by two lattice spacings in the [1-10] direction. The length and separation distance of these pieces along the [001] direction varied, but were mostly about two lattice spacings. These areas were highly dynamic and features were observed to shift position between STM scans. The holes were first observed at $\sim 3$ ML Co coverage (upper right corner of Fig. 9). In Fig. 11, with 10 ML Co coverage, the holes were $\sim 30 \AA$ in size. They were almost square in shape, but were slightly elongated along the [001] direction and $~ 3-6$ ML deeper than the surface. The structure at the bottom of the hole was not resolved. We speculate that the formation of these regions is associated with the balance of oxygen and cobalt content on the surface. Tölkes et al. reported that the (3 $\times 1)$ structure contains two oxygen atoms per unit cell, ${ }^{26}$ which is more than the oxygen content in the $(2 \times$ 1) of the original $\mathrm{Cu}-\mathrm{O}$ surface and the $(2 \times 2)$ structure at 1 ML Co. Transformation to the $(3 \times 1)$ structure would therefore result in oxygen deficiency. We believe that the less well-ordered regions and the holes were the oxygen deficient areas. To test this, we leaked oxygen $(0.6 \mathrm{~L})$ into the UHV system while imaging the 19 ML Co film. Fig. 14 shows an area of the surface away from the shadow of the tip during oxygen exposure. The area covered by the $(3 \times 1)$ structure had clearly increased. Moreover, the depth of the holes had decreased from $\sim 3-4$ ML to $\sim 2$ ML with the $(3 \times 1)$ structure appearing at the base. The additional oxygen had converted the oxygen-deficient area into the oxygen-rich $(3 \times 1)$ structure and resulted in a flatter and more homogeneous film.

The STM results above agree in general with the LEED and RHEED results. 3D clusters were formed

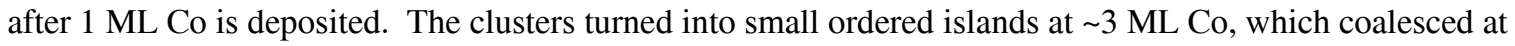
5 ML Co. Quasi layer-by-layer growth took place above this thickness. The surface was composed of (3 $\times 1)$ domains, together with some ill-defined area and had an average roughness of about three monolayers. Above 20 ML Co, edge dislocations form to relax the strain of the film. STM studies of Co film grown on clean $\mathrm{Cu}(110)$ at room temperature has been reported in the literature and anisotropic 3D islands have been found. ${ }^{12,15}$

\section{SUMMARY}


We have shown that oxygen as a surfactant greatly improved the growth of $\mathrm{Co}$ on $\mathrm{Cu}(110)$. The resulting Co film was continuous with a more or less flat and ordered surface, as opposed to the 3D islands found for $\mathrm{Co}$ grown on clean $\mathrm{Cu}(110)$. No $\mathrm{Cu}$ segregation was observed in the oxygen-assisted growth film. The difference in film quality affected the magnetic properties of the Co films. Although Co films grown with and without oxygen had the same easy axis along the [001] direction, the onset of magnetization at room temperature occurred at different thickness: Co films grown on clean $\mathrm{Cu}(110)$ had onset magnetization at $11 \mathrm{ML}$ of $\mathrm{Co}$, while the use of an oxygen surfactant reduced the onset magnetization thickness to $5 \mathrm{ML} \mathrm{Co}$, where the 3D islands coalesced into a continuous film. Our STM study revealed that the smoothing of the Co film by the oxygen surfactant was associated with various surface reconstructions at different thickness regimes, which most likely lowered the surface energy of the film.

\section{ACKNOWLEDGMENTS}

This work was supported by the U.S. Air Force, Office of Scientific Research Contract No. AFOSR-MIPR-95-0052 through the U.S. Department of Energy under Contract No. DE-AC03-76SF00098, and by National Science Foundation DMR-9805222. W.L. acknowledges the U.S. Department of Education for her research fellowship. 


\section{REFERENCES}

*Present address: Institute of Applied Physics, Tsukuba University, Ibraki 305-8577, Japan

${ }^{1}$ S. S. P. Parkin, N. More, and K. P. Roche, Phys. Rev. Lett. 64, 2304 (1990).

${ }^{2}$ M. N. Baibich, J. M. Broto, A. Fert, F. Nguyen Van Dau, F. Petroff, P. Etienne, G. Creuzet, A. Friederich, and J. Chazelas, Phys. Rev. Lett. 61, 2472 (1988).

${ }^{3}$ L. M. Falicov, D. T. Pierce, S. D. Bader, R. Gronsky, K. B. Hathaway, H. J. Hopster, D. N. Lambeth, S. S. P. Parkin, G. Prinz, M. Salamon, I. K. Schuller, and R. H. Victora, J. Mater. Res. 5, 1299 (1990).

${ }^{4}$ Y. Saito, K. Inomata, and K. Yusu, Phys. Rev. B 52, 6500 (1995).

${ }^{5}$ T. Thomson, P. C. Riedi, and D. Greig, Phys. Rev. B 50, 10319 (1994).

${ }^{6}$ J. Camarero, T. Graf, J. J. de Miquel, R. Miranda, W. Kuch, M. Zharnikov, A. Dittschar, C. M. Schneider, and J. Kirschner, Phys. Rev. Lett. 76, 4428 (1996).

${ }^{7}$ U. Bovensiepen, P. Poulopoulos, W. Platow, M. Farle, and K. Baberschke, J. Magn. Magn. Mater. 192, L 386 (1999).

${ }^{8}$ M. T. Keif and W. F. Egelhoff, Jr., Phys. Rev. B 47, 10785 (1993).

${ }^{9}$ R. K. Kawakami, E. J. Escorcia-Aparicio, and Z. Q. Qiu, Phys. Rev. Lett. 77, 2570 (1996).

${ }^{10}$ H. J. Choi, R. K. Kawakami, E. J. Escorcia-Aparicio, Z. Q. Qiu, J. Pearson, J. S. Jiang, D. Li, and S. D. Bader, Phys. Rev. Lett. 82, 1947 (1999).

${ }^{11}$ B. Hillebrands, J. Fassbender, R. Jungblut, G. Güntherodt, D. J. Roberts, and G. A. Gehring, Phys. Rev. B 53, R10548 (1996).

${ }^{12}$ J. Fassbender, G. Güntherodt, C. Mathieru, B. Hillebrands, R. Jungblut, J. Kohlhepp, M. T. Johnson, D. J. Roberts, and G. A. Gehring, Phys. Rev. B 57, 5870 (1998).

${ }^{13}$ S. Hope, E. Gu, M. Tselepi, M. E. Buckley, and J. A. C. Bland, Phys. Rev. B 57, 7454 (1998).

${ }^{14}$ S. Hope, E. Gu, B. Choi, and J. A. C. Bland, Phys. Rev. Lett. 80, 1750 (1998).

${ }^{15}$ S. Hope, M. Tselepi, E. Gu, T. M. Parker, and J. A. C. Bland, J. Appl. Phys. 85, 6094 (1999).

${ }^{16}$ M. Kim, L. Zhong, and A. J. Freeman, Phys. Rev. B 57, 5271 (1998). 
${ }^{17}$ M. Cinal, D. M. Edwards, and J. Mathon, J. Magn. Magn. Mater. 140-144, 681 (1995).

${ }^{18}$ G. Y. Guo, D. J. Roberts, and G. A. Gehring, Phys. Rev. B 59, 14466 (1999).

${ }^{19}$ A. V. Smirnov and A. M. Bratkovsky, Phys. Rev. B 55, 14434 (1997).

${ }^{20}$ C. Tölkes, R. Struck, R. David, P. Zeppenfeld, and G. Comsa, Phys. Rev. Lett. 80, 2877 (1998).

${ }^{21}$ W. Ling, O. Takeuchi, D. F. Ogletree, Z. Q. Qiu, and M. Salmeron, Surf. Sci. 450, 227 (2000).

${ }^{22}$ D. J. Coulman, J. Wintterlin, R. J. Behm, and G. Ertl, Phys. Rev. Lett. 64, 1761 (1990).

${ }^{23}$ Y. Kuk, F. M. Chua, P. J. Silverman, and J. A. Meyer, Phys. Rev. B 41, 12393 (1990).

${ }^{24}$ J. J. de Miguel, A. Cebollada, J. M. Gallego, R. Miranda, et al., J. Magn. Magn. Mat. 93, 1 (1991).

${ }^{25}$ P. Krams, F. Lauks, R. L. Stamps, B. Hillebrands, and G. Güntherodt, Phys. Rev. Lett. 69, 3674 (1992).

${ }^{26}$ C. Tölkes, R. Struck, R. David, P. Zeppenfeld, and G. Comsa, Appl. Phys. Lett. 73, 1059 (1998).

27 J. Zhong, E. Wang, Q. Niu, Z. Zhang, Phys. Rev. Lett. 84, 3895 (2000). 


\section{FIGURE CAPTIONS}

FIG. 1. Schematic of the Co wedge sample grown for LEED, RHEED and SMOKE measurements. A thick Co film was grown at the end of the wedge to register the wedge position.

FIG. 2. $\mathrm{Co}, \mathrm{Cu}$ and $\mathrm{O}$ Auger peak heights, as a function of $\mathrm{Co}$ thickness deposited on the $\mathrm{Cu}(110)-(2 \times$ 1)O surface at $350 \mathrm{~K}$. The continuous curves are guides to the eye.

FIG. 3. Longitudinal SMOKE loops with applied magnetic field along the [001] and [1-10] directions for $\mathrm{Co}$ films of various thicknesses grown on $\mathrm{Cu}(110)$ with $\mathrm{O}$ as a surfactant. The onset magnetization is at 5 ML Co, and the easy axis is along the [001] direction.

FIG. 4. Longitudinal SMOKE loops with applied magnetic field along the [001] and [1-10] directions for $\mathrm{Co}$ films of various thicknesses grown on clean $\mathrm{Cu}(110)$. The onset magnetization is at $~ 11$ ML Co, and the easy axis is along the [001] direction.

FIG. 5. Magnetic remanence of Co films grown on $\mathrm{Cu}(110)$ (a) with and (b) without $\mathrm{O}$ surfactant as a function of Co film thickness, with magnetic field applied along the [001] direction at room temperature. The two graphs are on the same scale - note the difference in the slopes of the two curves. A magnetic signal is detected starting from a much lower Co thickness for the film grown with $\mathrm{O}$ as a surfactant.

FIG. 6. (a) Evolution of LEED pattern for Co film with increasing thickness grown on $\mathrm{Cu}(110)$ with $\mathrm{O}$ as a surfactant. The electron beam energy was $104.8 \mathrm{eV}$. $0 \mathrm{ML}$ Co corresponds to the starting $\mathrm{Cu}(110)-(2$ $\times 1) \mathrm{O}$ surface. A weak $(1 \times 2)$ periodicity appears at 1 ML Co. The LEED pattern becomes increasingly

broad and diffuse until $\sim 5 \mathrm{ML}$ Co, at which point only the primary spots are visible. Above 5 ML Co, the pattern re-sharpens, with new spots corresponding to $2 \mathrm{x}$ and $3 \mathrm{x}$ periodicities along the [1-10] direction. (b) RHEED patterns reproduce the information extracted from LEED. The electron beam is parallel to the 
[001] direction.

FIG. 7. (a) Evolution of LEED pattern for Co film with increasing thickness grown on clean $\mathrm{Cu}(110)$. The electron beam energy was $105.4 \mathrm{eV}$. Co grows pseudomorphically, as indicated by the $\mathrm{p}(1 \times 1)$ pattern. However, in contrast with the Co films grown with $\mathrm{O}$ as a surfactant, the broad spots and diffused background show that the growth is 3D throughout the thickness range studied. Faint streaks, as well as spots showing weak surface reconstruction, are also visible for Co films of higher thickness (>20 ML). (b) RHEED patterns with the electron beam parallel to the [001] direction agree with the results from LEED.

FIG. 8. $350 \AA \times 350 \AA$ image of $1.7 \mathrm{ML}$ Co on $\mathrm{Cu}(110)$ with $\mathrm{O}$ as a surfactant. The tunneling current was $0.03 \mathrm{nA}$ and the tip bias voltage was $-0.5 \mathrm{~V}$. 3D clusters have covered the surface.

FIG. 9. $450 \AA \times 450 \AA$ image of $3 \mathrm{ML}$ Co on $\mathrm{Cu}(110)$ with $\mathrm{O}$ as a surfactant. The tunneling current was $0.48 \mathrm{nA}$ and the bias voltage was $-2 \mathrm{~V}$. The $3 \mathrm{D}$ clusters have transformed into rectangular islands with surface structure.

FIG. 10. $1000 \AA \times 1000 \AA$ image of 5 ML Co on $\mathrm{Cu}(110)$ with $\mathrm{O}$ as a surfactant. The tunneling current was $0.1 \mathrm{nA}$ and the bias voltage was $0.9 \mathrm{~V}$. The islands start to coalesce to form a continuous film.

FIG. 11. $500 \AA \times 500 \AA$ image of $10 \mathrm{ML}$ Co on $\mathrm{Cu}(110)$ with $\mathrm{O}$ as a surfactant. The tunneling current was $0.04 \mathrm{nA}$ and the bias voltage was $-0.2 \mathrm{~V} .(3 \times 1)$ patches can be identified in some areas.

FIG. 12. $300 \AA \times 300 \AA$ image of $19 \mathrm{ML}$ Co on $\mathrm{Cu}(110)$ with $\mathrm{O}$ as a surfactant. The tunneling current was $0.5 \mathrm{nA}$ and the bias voltage was $-1.6 \mathrm{~V}$. $(3 \times 1)$ patches are more clearly visible.

FIG. 13. $2000 \AA \times 2000 \AA$ image of 24 ML Co on $\mathrm{Cu}(110)$ with O surfactant. The tunneling current was $0.1 \mathrm{nA}$ and the bias voltage was $1.4 \mathrm{~V}$. A number of edge dislocations (lines along the [1-10] 
direction) are formed to relax the strain of the film.

FIG. 14. $400 \AA \times 400 \AA$ image of 19 ML Co on $\mathrm{Cu}(110)$ with O as a surfactant, after exposure to 0.6 $\mathrm{L}$ of oxygen. The tunneling current was $0.7 \mathrm{nA}$ and the bias voltage was $-1.1 \mathrm{~V}$. The area with $(3 \times 1)$ reconstruction has substantially increased. Moreover, the bottom of the holes can now be resolved and the holes also have the $(3 \times 1)$ structure after oxygen dosage. 